\title{
The Effects of Sa-Am Acupuncture on the Radial Pulse in Healthy Human Subjects: A Comparative Study of Pericardium jung-gyuck and Triple Energizer jung-gyuck
}

\author{
Tae-Min Kim, Jea-Hong Kim, Hyun-Suk Lee, Yun-Kyoung Yim \\ Department of Meridian and Acupoint, College of Korean Medicine, Daejeon University, Daejeon, Korea
}

\begin{abstract}
Objectives: The purpose of this study is to investigate the effects of Sa-am acupuncture with Pericardium jung-gyuck (SA-acu-PC) and Triple Energizer jung-gyuck (SA-acu-TE) on the radial pulse in healthy human subjects. Methods: Sixty healthy subjects participated in this study and divided into three groups randomly: Control group(C), Pericardium jung-gyuck group(PC), Triple Energizer jung-gyuck group(TE). Radial pulse was measured by 3 dimensional pulse imaging system (DMP-3000) before, right after, 30 minutes after, and 60 minutes after acupuncture. Results: 1 . The changes observed in periodic parameters are considered as the common effects of both SA-acu-TE and SA-acu-PC. SA-acu-TE was more effective than SA-acu-PC in these effects.

2. The effects of SA-acu-PC appeared in the pulse amplitude, pulse area, and pulse power volume mainly at the left chon position, and

3. The effects of SA-acu-TE appeared in the radial augmentation index and main peak angle mainly at the right gwan position.

Conclusions: The effects of SA-acu-PC and SA-acu-TE can be observed in the radial pulse. The increases in pulse amplitude, pulse area, and pulse power volume at the left chon may imply the effects of SA-acu-PC. And the decrease in the pulse power volume, the increases in radial augmentation index and main peak angle may imply the effects of SA-acu-TE.
\end{abstract}

$\overline{\text { Key Words }}$ : Radial pulse, Sa-am acupuncture, Pericardium jung-gyuck, Triple Energizer jung-gyuck.

\section{Introduction}

In Oriental medical diagnosis, the radial pulse is an important indicator of the conditions of internal organs and meridians. The pulse is examined at three different positions on the wrist, which are called chon, gwan, and cheock, bilaterally. Each position is considered to indicate the states of the corresponding organs ${ }^{1)}$.

According to Oriental medical physiology, a Yin organ and a Yang organ with the same five-phases property are coupled. This coupling is called the Yin-Yang exterior-interior relationship. The pericardium and the triple energizer are in a Yin-Yang exterior -interior relationship ${ }^{2)}$.

The pericardium is the outer shield of the heart. It protects the heart, prevents evil factors from infiltrating the heart, and helps spiritual stability). The triple energizer is also known as the triple burner, triple warmer, or triple heater. Visceral qi

\footnotetext{
- Received : 6 December 2017

- Revised : 21 December 2017

- Accepted : 21 December 2017

- Correspondence to: Yun-Kyoung Yim

Department of Meridian and Acupoint, College of Korean Medicine, Daejeon University

62, Daehak-ro, Dong-gu, Daejeon 300-716, Korea

Tel : +82-42-280-2610, Fax : +82-42-280-2641, E-mail : docwindy@dju.kr
} 
transformation and water movement are regulated by the triple energizer ${ }^{2}$.

The pericardium and the triple energizer are coupled and have many features in common. For example, they are both involved in the movement of ministerial fire. However, their functions are opposed in some respects. For example, the pericardium supports blood circulation, while the triple energizer regulates qi transformation ${ }^{4}$.

Acupuncture is a therapeutic method that stimulates certain points on the body surface, most commonly with fine needles, in order to treat or prevent diseases. Sa-Am acupuncture is a kind of acupuncture technique initiated by a Korean physician named Sa-Am. This technique is characterized by the application of the five-phases theory and the mother-child reinforcement-reduction principle to the selection of points and needle manipulation ${ }^{5)}$.

The specific technique of Sa-Am acupuncture for reinforcing the function of the corresponding organ and meridian is called jung-gyuck ${ }^{6}$. For instance, the pericardium jung-gyuck reinforces the function of the pericardium in activating the cardiovascular system and improving blood circulation. The triple energizer jung-gyuck reinforces the function of the triple energizer in activating qi transformation and in regulating the waterways ${ }^{2}$.

Pulse is affected by various factors, and is also known to be altered by acupuncture. Previous studies have shown that acupuncture induces immediate changes in pulse, and the results vary depending on the points used and the techniques applied ${ }^{7-11}$. Acupuncture influences the functions of internal organs and meridians, and the pulse indicates the physiological and pathological conditions of the body. So it can be hypothesized that the functions of the pericardium and the triple energizer will be reinforced by $\mathrm{Sa}-\mathrm{Am}$ acupuncture with pericardium jung-gyuck (SA-acu-PC), and Sa-Am acupuncture with triple energizer jung-gyuck (SA-acu-TE), respectively, and consequently that the common aspects as well as the opposite aspects of the actions of SA-acu-PC and SA-acu-TE may be reflected in the pulse.

To investigate the effects of SA-acu-PC and SA-acu-TE on pulse, sixty healthy volunteers participated in this study, and their pulses were measured before and after acupuncture.

\section{Methods}

\section{Subjects}

Sixty healthy volunteers with no underlying diseases or prescribed medicines were randomly divided into three groups: control group, Sa-Am acupuncture group with triple energizer jung-gyuck (TE group), and Sa-Am acupuncture group with pericardium jung-gyuck (PC group).

The criteria for exclusion were as follows: arrhythmia, systolic blood pressure $>150$, diastolic blood pressure $<60$, wounds or scars in the region of pulse measurement, body mass index (BMI) $<18$ $\mathrm{kg} / \mathrm{m}^{2}$ or BMI $>32 \mathrm{~kg} / \mathrm{m}^{2}$, pregnancy, women in menstruation.

All subjects provided written informed consents. This study was approved by the institutional review board of Daejeon Korean Hospital of DaeJeon University (approval no. P2012-4).

\section{Experimental Procedure}

The experimental procedure is presented in Figure 1. All subjects took 30 minutes of rest sitting on a comfortable chair before the experiment. The radial pulse was measured in a sitting position. After the first measurement of the radial pulse, the participants in the acupuncture groups (TE and PC groups) were given acupuncture in the supine position for 30 minutes, and the participants in the control group took 30 minutes of rest in bed. After acupuncture or bed rest, the radial pulse was measured again in the 


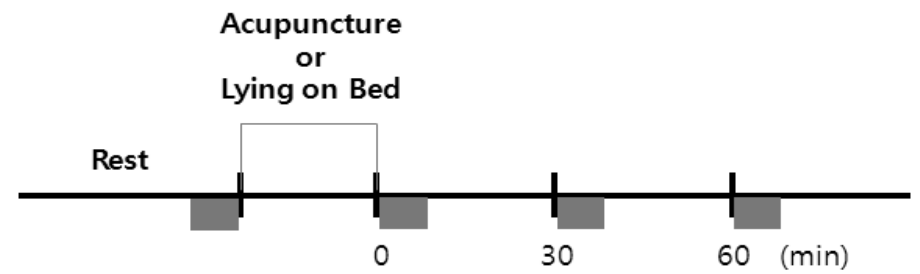

Pulse Mesurement

Fig. 1. Experimental protocol

Table 1. Acupoints and Manipulation Techniques of PC and TE Jung-gyuck

\begin{tabular}{|c|c|c|}
\hline \multirow{2}{*}{ Sa-Am acupuncture } & \multicolumn{2}{|c|}{ Acupoints and Manipulation } \\
\hline & Reinforcing & Reducing \\
\hline \multirow{2}{*}{ PC jung-gyuck } & PC9 & PC3 \\
\hline & LR1 & $\mathrm{KI} 10$ \\
\hline \multirow{2}{*}{ TE jung-gyuck } & TE3 & TE2 \\
\hline & GB41 & BL66 \\
\hline
\end{tabular}

same manner as the first measurement, taking three measurements with 30-minute intervals. The participants were asked to remain calm throughout the experiment. Participants were allowed water, but were restricted from consuming food or other drinks. The experimental room was kept at a stable temperature $\left(24-26{ }^{\circ} \mathrm{C}\right)$ and humidity (40-60\%).

\section{Acupuncture}

Acupuncture was performed by one Korean Medical Doctor using disposable needles $(0.25 \times 30 \mathrm{~mm}$, Dong Bang Acupuncture Co. Korea). The acupoints used in the acupuncture groups are presented in Table 1. PC9 was located 0.1 cun proximally from the radial corner of the middle fingernail, on the intersection of the vertical line of the radial side of the middle fingernail and the horizontal line of the base of the middle fingernail. The other points were located according to WHO standard. All points were used bilaterally. Reinforcing manipulation was done by inserting the needles obliquely following the meridian flow, and rotating the needles clockwise.
On the contrary, reducing manipulation was done by inserting the needles obliquely against the meridian flow, and rotating the needles counterclockwise ${ }^{13)}$. The total acupuncture procedure including insertion, manipulation, retention and withdrawal of the needles took 30 minutes.

\section{Pulse Measurement and Data acquisition}

The radial pulse was measured at six different positions (bilateral chon, gwan and cheock) on the wrist. For reliable data acquisition and experimental reproducibility, the position of Gwan was marked on the skin in the first measurement. The next measurements were conducted based on this mark, thus guaranteeing that the pulse was measured at the same positions in every measurement.

The pulse was measured using a multi-step tonometry system (DMP-3000, Daeyomedi Co. Ltd., Korea). This device has an arterial tonometry sensor with an array of five piezoresistive semiconductor transducers applying multiple levels of pressure to obtain stable multiple-step pulse waveforms. 
Table 2. STandards for Reporting Interventions in Clinical Trials of Acupuncture (STRICTA) 2010 Checklist of Information to include when Reporting Interventions in a Clinical Trial of Acupuncture ${ }^{12)}$.

\begin{tabular}{|c|c|}
\hline Item & Detail \\
\hline \multirow{3}{*}{ 1. Acupuncture Rationale } & 1a) Style of acupuncture : Sa-am acupuncture \\
\hline & 1b) Literature sources to justify rationale : Practice of Acupuncture; A Guidebook for College Students ${ }^{13)}$ \\
\hline & 1c) Extent to which treatment was varied : none \\
\hline \multirow[t]{7}{*}{ 2. Details of Needling } & 2a) Number of needle insertions per subject per session (mean and range where relevant) $: 8$ \\
\hline & $2 \mathrm{~b}$ ) Names (or location if no standard name) of points used (uni/bilateral) : Presented in Table 1(Bilateral) \\
\hline & 2c) Depth of insertion, based on a specified unit of measurement, or on a particular tissue level : $2 \sim 14 \mathrm{~mm}$ \\
\hline & 2d) Response sought (e.g. de qi or muscle twitch response) : Obtaining qi \\
\hline & $\begin{array}{l}\text { 2e) Needle stimulation (e.g. manual, electrical) : twirling supplementation and draining method, directional } \\
\text { supplementation and draining method }\end{array}$ \\
\hline & 2f) Needle retention time $: 30 \mathrm{~min} \quad w$ \\
\hline & $\begin{array}{l}\text { 2g) Needle type (diameter, length, and manufacturer or material) : } 0.25 \times 30 \mathrm{~mm} \text {, Dong Bang Acupuncture Co. } \\
\text { Korea }\end{array}$ \\
\hline \multirow{2}{*}{ 3. Treatment Regimen } & 3a) Number of treatment sessions : 1 \\
\hline & 3b) Frequency and duration of treatment sessions : 1 \\
\hline \multirow{2}{*}{$\begin{array}{l}\text { 4. Other Components of } \\
\text { Treatment }\end{array}$} & $\begin{array}{l}\text { 4a) Details of other interventions administered to the acupuncture group (e.g. moxibustion, cupping, herbs, } \\
\text { exercises, lifestyle advice) : none }\end{array}$ \\
\hline & $\begin{array}{l}\text { 4b) Setting and context of treatment, including instructions to practitioners, and information and } \\
\text { explanations to patients : none }\end{array}$ \\
\hline 5. Practitioner Background & $\begin{array}{l}\text { (5) Description of participating acupuncturists (qualification or professional affiliation, years in } \\
\text { acupuncture practice, other relevant experience) : Korean medical doctor with five years' experience. }\end{array}$ \\
\hline \multirow{2}{*}{$\begin{array}{l}\text { 6. Control and Comparator } \\
\text { Intervention }\end{array}$} & $\begin{array}{l}\text { 6a) Rationale for the control or comparator in the context of the research question, with sources that justify } \\
\text { this choice : According to the previous study, short-term bed rest induces significant changes in the } \\
\text { radial pulse. The participants in the control group took a rest in bed for } 30 \text { min, since the participants in } \\
\text { the acupuncture groups (TE and PC groups) were given acupuncture in bed for } 30 \text { min }^{16)}\end{array}$ \\
\hline & $\begin{array}{l}\text { 6b) Precise description of the control or comparator. If sham acupuncture or any other type of } \\
\text { acupuncture-like control is used, provide details as for Items } 1 \text { to } 3 \text { above. : The participants were } \\
\text { restricted from consuming food or other drinks except water }\end{array}$ \\
\hline
\end{tabular}

In this study, the pressure was applied at five different levels $(50 \mathrm{~g}, 90 \mathrm{~g}, 140 \mathrm{~g}, 190 \mathrm{~g}$ and 240 $\mathrm{g}$ ), and the pulse waves were recorded for five seconds at each level. The data at each level included five pulse waves from five piezoresistive semiconductor transducers. Therefore, 25 pulse waves were obtained from each measuring position.

\section{Data analysis}

1) Pulse wave selection

Among the five different pulse waves obtained at five different levels of applied pressure, the pulse waveform with the highest main peak was the most distinct and suitable to observe the pulse characteristics; therefore, it was selected as the 'representative pulse wave' (Figure 2).

The array sensor has five (medial, lateral, distal, proximal, and central) transducers. Among the five transducers, the central transducer is placed above the center of arterial flow. Therefore, the representative pulse wave from the central transducer was mainly analysed in this study.

\section{2) Pulse parameters}

The pulse parameters were gained by time domain analysis of pulse wave (Figure 3). Pulse power volume was estimated with the data from all of the five piezoresistive semiconductor transducers. The other parameters were obtained through the data only from the central transducer. 


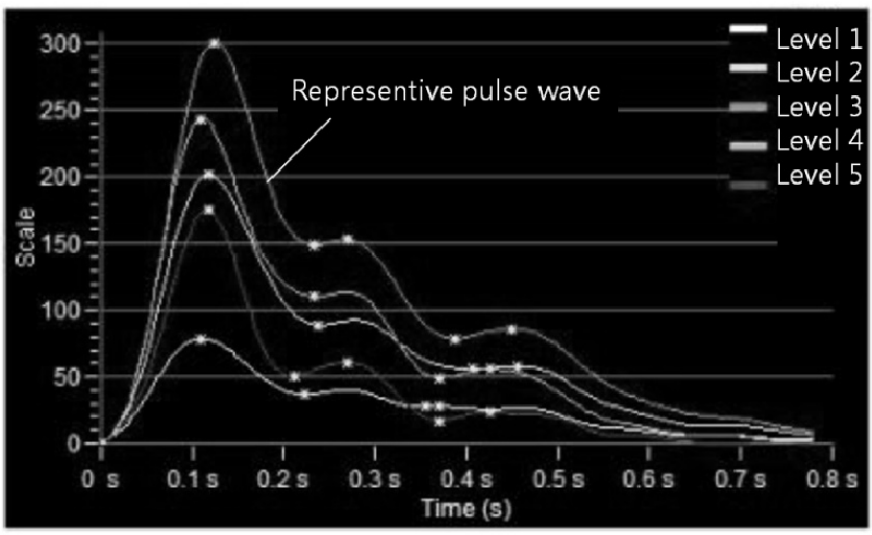

Fig. 2. Radial pulse waveforms obtained with 5 levels of applied pressure Scale on $y$-axis means div (digital value for pressure).

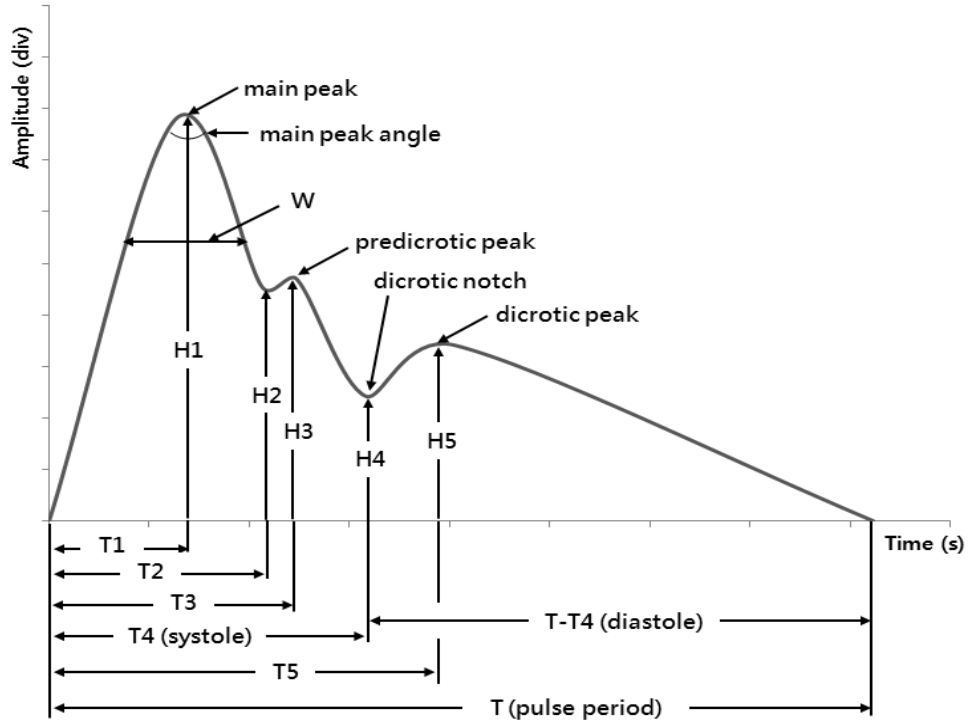

Fig. 3. Radial pulse waveform

(1) $\mathrm{T}$ : total pulse period (s)

(2) $\mathrm{T} 4 / \mathrm{T}$ : rate of systolic pulse period to total pulse period

(3) (T-T4)/T: rate of diastolic pulse period to total pulse period

(4) $\mathrm{H} 1$ : Amplitude of main peak

(5) H3 : Amplitude of predicrotic peak
(6) RAI (Radial Augmentation Index) : H3/H1

(7) $\mathrm{W}$ (high-tensioned pulse period) : width of main peak where the pulse height is $2 / 3$ of $\mathrm{H} 1(\mathrm{~s})$

(8) W/T : rate of high-tensioned pulse period to total pulse period

(9) MPA (Main Peak Angle) : angle of main peak 
Table 3. General Characteristics of Subjects

\begin{tabular}{cccc}
\hline & Control $(\mathrm{n}=20)$ & PC $(\mathrm{n}=20)$ & TE $(\mathrm{n}=20)$ \\
\cline { 2 - 4 } & Mean \pm SD & Mean \pm SD & Mean \pm SD \\
\hline Age(year) & $21.00 \pm 2.17$ & $23.86 \pm 2.97$ & $23.61 \pm 3.38$ \\
Height $(\mathrm{cm})$ & $173.17 \pm 4.23$ & $173.95 \pm 5.44$ & $173.17 \pm 4.23$ \\
Weight $(\mathrm{kg})$ & $67.83 \pm 8.91$ & $66.62 \pm 8.16$ & $67.83 \pm 8.91$ \\
BMI & $22.61 \pm 2.85$ & $22.00 \pm 2.31$ & $22.61 \pm 2.85$ \\
\hline
\end{tabular}

Values are presented as mean \pm SD (standard deviations). PC : Sa-Am acupuncture group with pericardium jung-gyuck. TE : Sa-Am acupuncture group with triple energizer jung-gyuck

of pulse wave

(10) Pulse Area : total pulse area estimated with pulse wave $\left(\operatorname{div}^{2}\right)$

(11) Pulse Power volume : value estimated by integration of highest amplitudes of five representative pulses from the five transducers of the sensor $\left(\mathrm{div}^{3}\right)$

\section{3) Statistical analysis}

The statistical analysis was performed using PASW Statistics 18.0 (IBM, USA). The data of the control group were used as the baseline to analyze the effect of acupuncture on pulse parameters. The changes of each parameters were analysed using repeated measures of analysis of variance (ANOVA). Inter-group comparisons at each time point were analysed using one-way ANOVA and least significant difference (LSD) tests or Mann-Whitney $\mathrm{U}$ test. $\mathrm{P}$ values of less than 0.05 were considered statistically significant.

\section{Results}

\section{Participants' General Characteristics}

There were no significant differences in age, height, weight, BMI (Table 3), and the baselines of pulse parameters (data not shown) between the groups.

\section{Periodic Parameters}

\section{1) Pulse Period}

The pulse period increased significantly at 0,30 , and $60 \mathrm{~min}$ in the TE group compared to the control group. It also increased significantly at $0 \mathrm{~min}$ in the PC group compared to the control group.

When compared between the PC group and the TE group, the pulse period increased significantly in the TE group compared to the $\mathrm{PC}$ group at 0,30 and 60min after acupuncture (Figure 4).

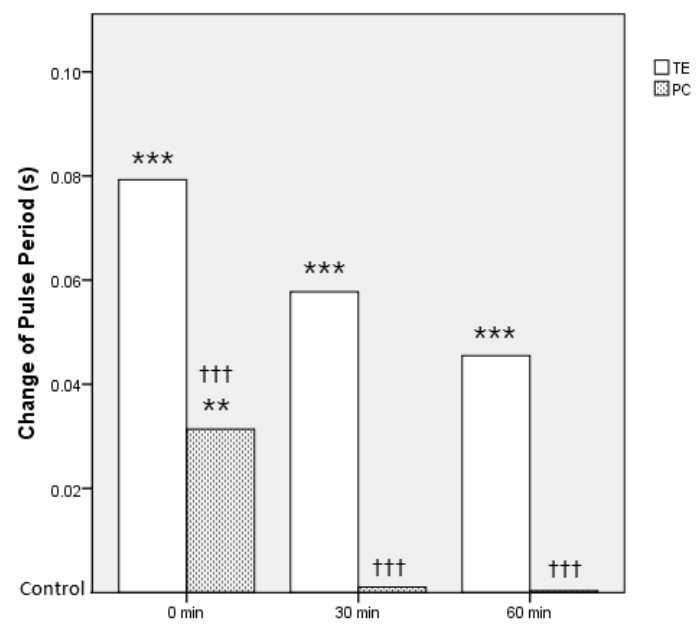

Fig. 4. Effects of Sa-Am Acupuncture with Pericardium Jung-gyuck and triple Energizer Jung-gyuck on Pulse Period ( $\mathrm{T}$ )

Data were expressed as mean White column: TE group, Gray column: PC group.

***: $p<0.001,{ }^{* *}$ : $p<0.01$ vs control

$\dagger \dagger \dagger: p<0.001$ vs TE 


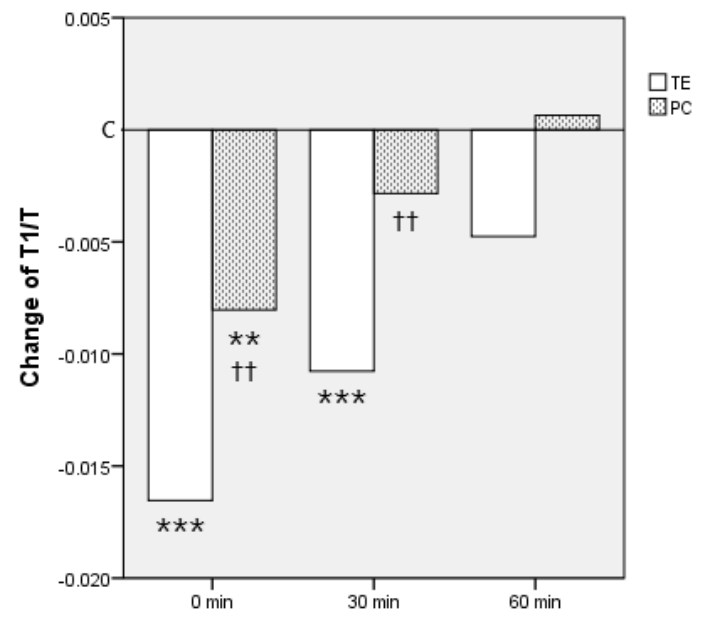

Fig. 5. Effects of Sa-Am Acupuncture with Pericardium Jung-gyuck and triple Energizer Jung-gyuck on $\mathrm{T} 1 / \mathrm{T}$

Data were expressed as mean.

C: control group, White column: TE group, Gray column: PC group. ***: $p\left\langle 0.001,{ }^{* *}: p<0.01\right.$ vs control

$\dagger \dagger: p<0.01$ vs TE

\section{2) $\mathrm{T} 1 / \mathrm{T}$}

$\mathrm{T} 1 / \mathrm{T}$ decreased significantly at 0 and $30 \mathrm{~min}$ in the TE group compared to the control group. It also decreased significantly at $0 \mathrm{~min}$ in the $\mathrm{PC}$ group compared to the control group.

When compared between the PC group and the TE group, T1/T decreased significantly in the TE group compared to the $\mathrm{PC}$ group at 0 and $30 \mathrm{~min}$ after acupuncture (Figure 5).

\section{3) $T 4 / T$}

$\mathrm{T} 4 / \mathrm{T}$ decreased significantly at 0,30 , and $60 \mathrm{~min}$ in the TE group compared to the control and the PC groups (Figure 6).

\section{4) $(\mathrm{T}-\mathrm{T} 4) / \mathrm{T}$}

(T-T4)/T increased significantly at 0,30 , and 60 min in the TE group compared to the control and PC groups (Figure 7).

5) $\mathrm{W} / \mathrm{T}$

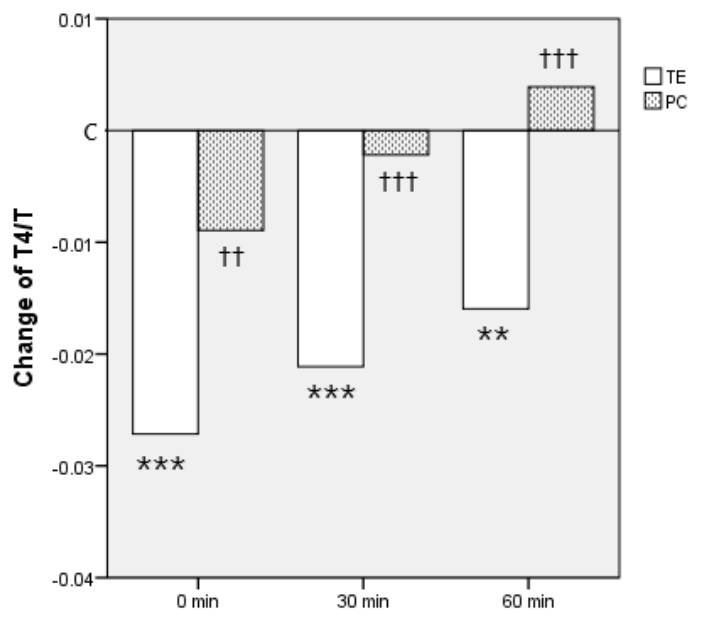

Fig. 6. Effects of Sa-Am Acupuncture with Pericardium Jung-gyuck and triple Energizer Jung-gyuck on $\mathrm{T} 4 / \mathrm{T}$

Data were expressed as mean.

C: control group, White column: TE group, Gray column: PC group. ***: $p<0.001,{ }^{* *}: p<0.01$ vs control

$\dagger \dagger \dagger: p<0.001, \dagger \dagger: p<0.01$ vs TE

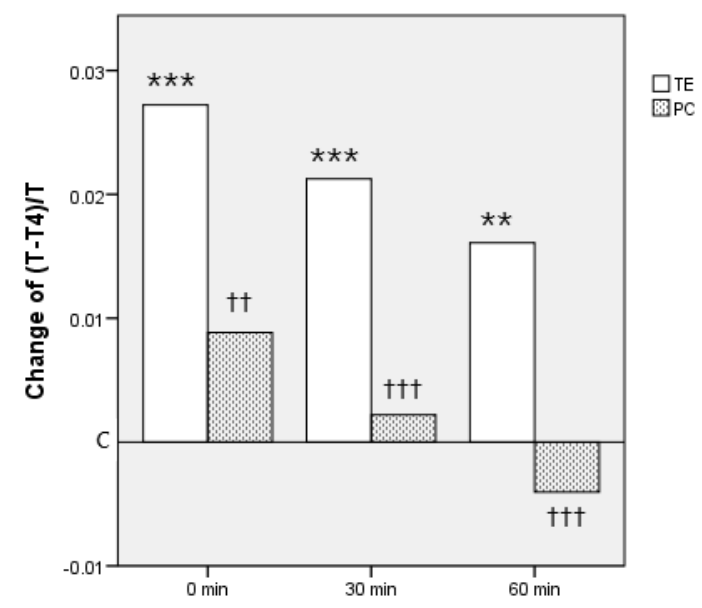

Fig. 7. Effects of Sa-Am Acupuncture with Pericardium Jung-gyuck and triple Energizer Jung-gyuck on $(\mathrm{T}-\mathrm{T} 4) / \mathrm{T}$

Data were expressed as mean.

C: control group, White column: TE group, Gray column: PC group. ***: $p<0.001,{ }^{* *}: p<0.01$ vs control

$\dagger \dagger \dagger: p<0.001, \dagger \dagger: p<0.01$ vs TE

W/T decreased significantly at $0 \mathrm{~min}$ in the TE group compared to the control and the PC groups 


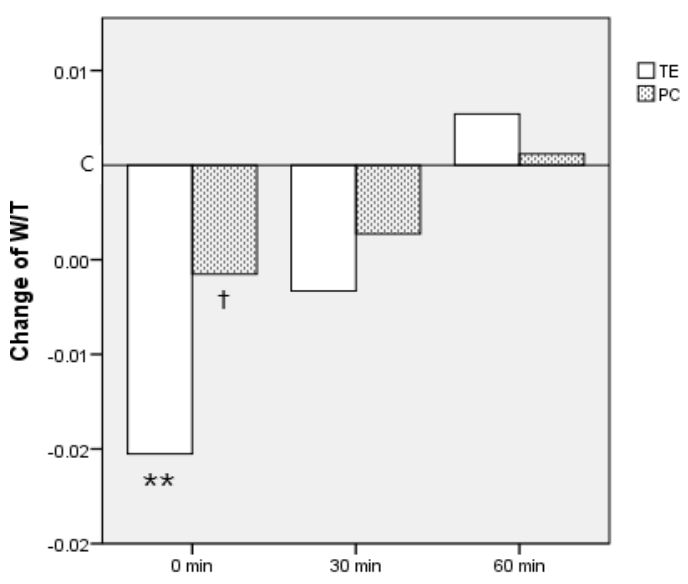

Fig. 8. Effects of Sa-Am Acupuncture with Pericardium Jung-gyuck and triple Energizer Jung-gyuck on W/T

Data were expressed as mean.

C: control group, White column: TE group, Gray column: PC group.

**: $p\langle 0.01$ vs control

$\dagger: p<0.05$ vs TE

(Figure 8).

\section{Pulse Amplitude}

The pulse amplitude of the PC group increased significantly at $0 \mathrm{~min}$ on $\mathrm{L} 1$ position, at $60 \mathrm{~min}$ on L1 and L2 positions compared to that of the TE group (Figure 9).

\section{Pulse Area}

Pulse area increased significantly at $0 \mathrm{~min}$ on L1 position in the PC group compared to the control group.

When compared between the PC group and the TE group, the total pulse area of the PC group increased significantly at 0 and $60 \mathrm{~min}$ on L1 position compared to that of the TE group (Figure $10)$.

\section{Pulse Power Volume}

The pulse power volume decreased significantly at 0 and $30 \mathrm{~min}$ on $\mathrm{R} 1$ position, at $0 \mathrm{~min}$ on $\mathrm{R} 2$
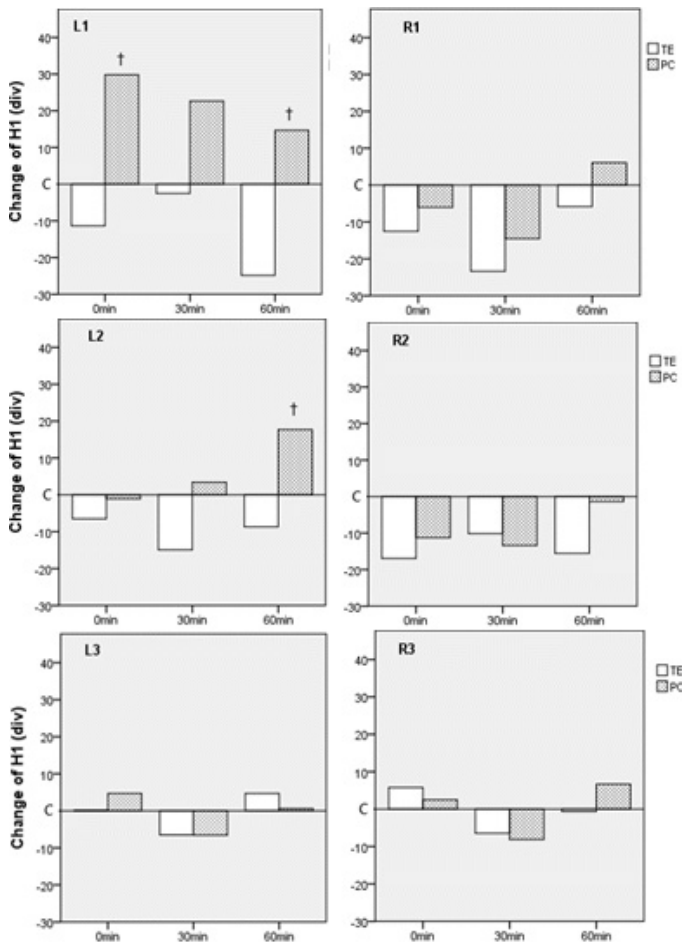

Fig. 9. Effects of Sa-Am Acupuncture with Pericardium Jung-gyuck and triple Energizer Jung-gyuck on Pulse Amplitude at each Measuring Position

Change in $\mathrm{H} 1$ at each time point on different measuring location was analyzed. Data were expressed as mean.

L1: left-Chon, L2: left-Gwan, L3: left-Cheock, R1: right-Chon, R2: right-Gwan, R3: right-Cheock.

C: control group, White column: TE group, Gray column: PC group. $\dagger: p<0.05$ vs TE

position in the TE group compared to the control group.

When compared between the PC group and the TE group, the pulse power volume of the PC group increased significantly at 0 and $60 \mathrm{~min}$ on L1 position compared to that of the TE group (Figure 11).

\section{Main Peak Angle}

Main peak angle increased significantly at 0 and 60 min on R2 position in TE group compared to the control group.

When compared between the PC group and the 

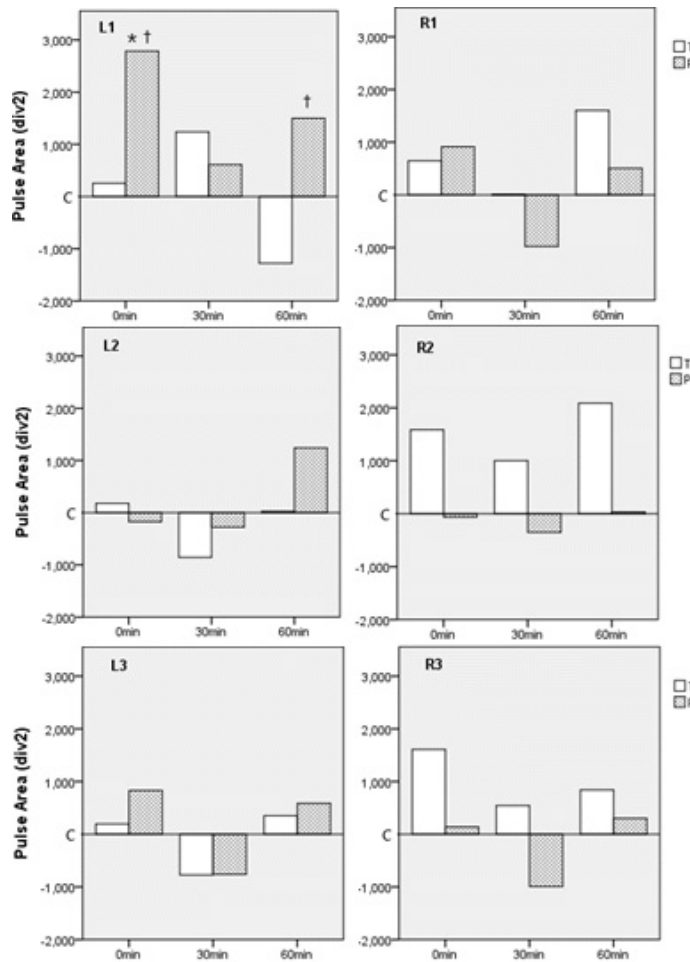

Fig. 10. Effects of Sa-Am Acupuncture with Pericardium Jung-gyuck and triple Energizer Jung-gyuck on Pulse Area at each Measuring Position

Change in the total pulse area at each time point on different measuring position was analyzed. Data were expressed as mean. L1: left-Chon, L2: left-Gwan, L3: left-Cheock, R1: right-Chon, R2: right-Gwan, R3: right-Cheock.

C: control group, White column: TE group, Gray column: PC group.

*: $p<0.05$ vs control

$\dagger: p<0.05$ vs TE

TE group, the main peak angle of TE group also increased significantly at 60 min compared to that of the PC group (Figure 12).

\section{Radial Augmentation Index}

Radial Augmentation Index (RAI) increased significantly at 0 and $60 \mathrm{~min}$ on $\mathrm{R} 2$ position in the TE group compared to the control group.

When compared between the PC group and the TE group, the RAI of the TE group increased significantly at $0 \mathrm{~min}$ on $\mathrm{R} 2$ position compared to that of the PC group (Figure 13).
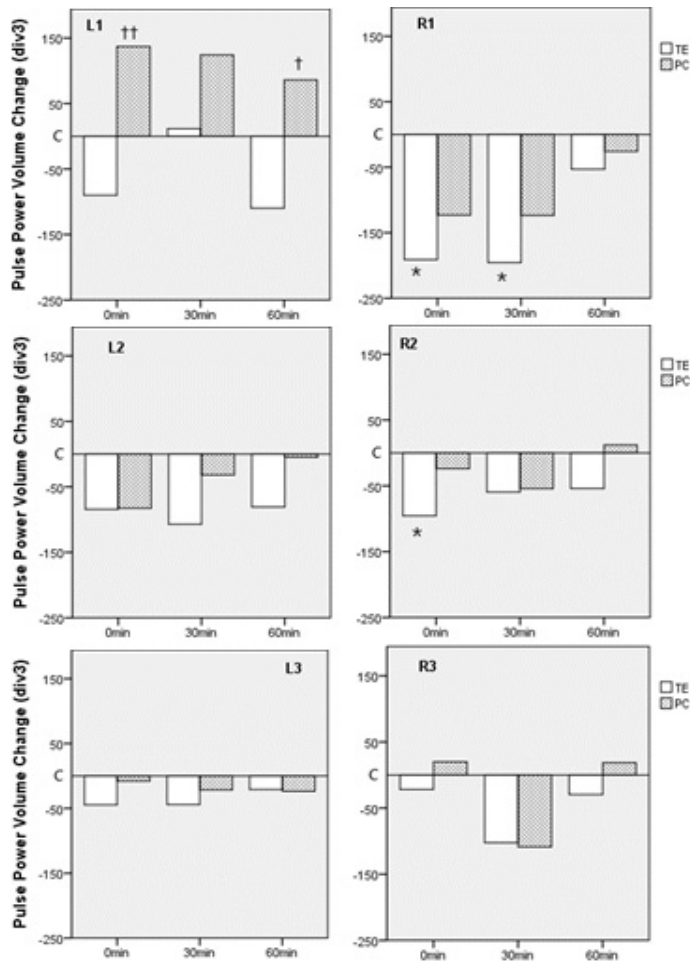

Fig. 11. Effects of Sa-Am Acupuncture with Pericardium Jung-gyuck and triple Energizer Jung-gyuck on Pulse Power Volume at each Measuring Position Change in the pulse power volume at each time point on different measuring position was analyzed. Data were expressed as mean.

L1: left-Chon, L2: left-Gwan, L3: left-Cheock, R1: right-Chon, R2: right-Gwan, R3: right-Cheock.

C: control group, White column: TE group, Gray column: PC group.

*: $p<0.05$ vs control

$\dagger \dagger: p<0.01, \dagger: p<0.05$ vs TE

\section{Discussion}

In Oriental medical pulse diagnosis, the radial pulse is taken at three different positions on each wrist, and the distal position is called chon, the proximal position is called cheock, and the middle position is called gwan. In terms of the theory of pulse diagnosis, the left-chon corresponds to the heart, the left-gwan to the liver, the left-cheock to the left kidney (kidney yin), the right-chon to the lung, the right-gwan to the spleen, and the 

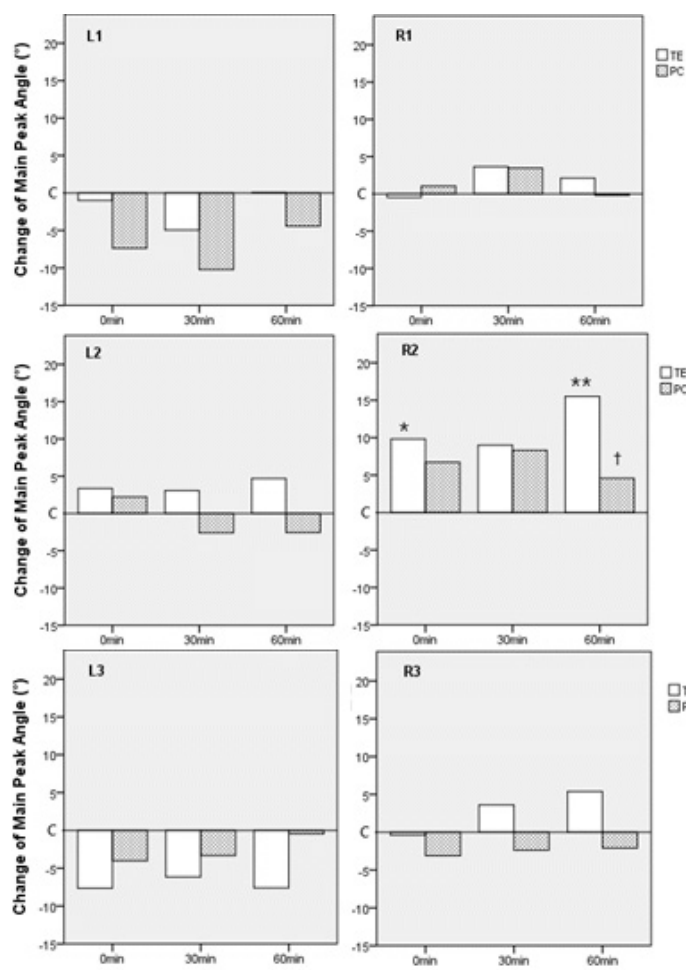

Fig. 12. Effects of Sa-Am Acupuncture with Pericardium Jung-gyuck and triple Energizer Jung-gyuck on Main Peak Angle at each Measuring Position

Change in the main peak angle at each time point on different measuring position was analyzed. Data were expressed as mean. L1: left-Chon, L2: left-Gwan, L3: left-Cheock, R1: right-Chon, R2: right-Gwan, R3: right-Cheock.

C: control group, White column: TE group, Gray column: PC group.

**: $p\langle 0.01, *: p<0.05$ vs control

$\dagger: p<0.05$ vs TE

right-cheock to the right kidney (kidney yang and life gate) ${ }^{14)}$.

In general, the organs located in the upper body correspond to the distal positions, and those in the lower body to the proximal positions. And the pulse is also examined at different levels of depth. The superficial level correlates with the Yang organs, while the deep level correlates with their coupled Yin organs ${ }^{1)}$.

In this study, Sa-Am acupuncture with pericardium jung-gyuck (SA-acu-PC), and Sa-Am acupuncture with triple energizer jung-gyuck (SA-acu-TE) were
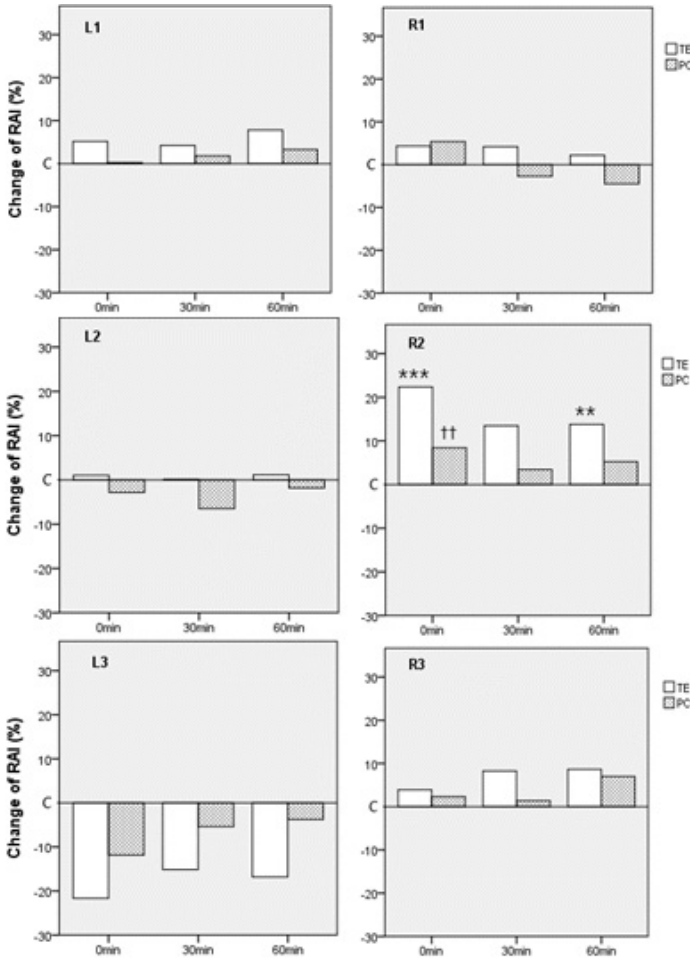

Fig. 13. Effects of Sa-Am Acupuncture with Pericardium Jung-gyuck and triple Energizer Jung-gyuck on RAl at each Measuring Position

Change in RAl at each time point on different measuring position was analyzed. Data were expressed as mean.

L1: left-Chon, L2: left-Gwan, L3: left-Cheock, R1: right-Chon, R2: right-Gwan, R3: right-Cheock.

C: control group, White column: TE group, Gray column: PC group. ${ }^{* * *}: p<0.001,{ }^{* *}: p<0.01$ vs control

$\dagger \dagger: p<0.01$ vs TE

administered and the changes in pulse parameters were analyzed.

The results show that the pulse period increased in both acupuncture groups (the PC group and the TE group) compared to the control group, indicating that heart rate was decreased by both SA-acu-PC and SA-acu-TE (Figure 4). (T-T4)/T represents the rate of diastolic pulse period to total pulse period, which also increased in both the TE and the PC groups compared to the control group (Figure 7). On the other hand, T1/T, W/T, and T4/T decreased in both acupuncture groups compared to the control group 
(Figures 5, 6, 8). T1/T is related to cardiovascular function, and a decrease in $\mathrm{T} 1 / \mathrm{T}$ indicates an increase in myocardial contractility ${ }^{15)}$. W/T is the rate of high-tensioned pulse period to total pulse period, and $\mathrm{T} 4 / \mathrm{T}$ is the rate of systolic pulse period to total pulse period.

Yim et al. reported that 30 minutes of bed-rest induced increases in $\mathrm{T}$ and (T-T4)/T and decreases in $\mathrm{T} 1 / \mathrm{T}, \mathrm{W} / \mathrm{T}$ and $\mathrm{T} 4 / \mathrm{T}^{16)}$. In the present study, the subjects received acupuncture for 30 minutes lying on a bed as in the study by Yim et al. All the changes of the periodic parameters of $\mathrm{T}, \mathrm{T} 1 / \mathrm{T}, \mathrm{W} / \mathrm{T}$, $\mathrm{T} 4 / \mathrm{T}$ and (T-T4)/T in the present study were greater in extent in the TE group than the $\mathrm{PC}$ group (Figures 4 - 8).

It is therefore postulated that SA-acu-PC, SA-acu-TE and bed-rest acted on these periodic parameters in the same direction. In other words, SA-acu-PC, SA-acu-TE and bed-rest all reduced heart rate, increased myocardial contractility, decreased the proportion of systole and high-tensioned pulse period, and increased the proportion of diastole. It may therefore be concluded that SA-acu-PC and SA-acu-TE strengthened the effects of bed-rest. Furthermore, the increases in $\mathrm{T}$ and $(\mathrm{T}-\mathrm{T} 4) / \mathrm{T}$, and the decrease in $\mathrm{T} 1 / \mathrm{T}, \mathrm{W} / \mathrm{T}$ and $\mathrm{T} 4 / \mathrm{T}$ can be considered as common effects of both SA-acu-TE and SA-acu-PC. SA-acu-TE was more effective than SA-acu-PC in respect of these parameters.

Parameters for pulse amplitude and waveform were analyzed at each measuring position. $\mathrm{H} 1$ is the amplitude of the main peak of the pulse wave. The pulse area is the two-dimensionally estimated value of the area formed with the pulse wave. The pulse power volume is the three-dimensionally estimated value of the volume formed by the amplitudes of the representative pulse waves from the five transducers of the sensor. H1, pulse area, and pulse power volume are known to be closely related ${ }^{8)}$.

In the present study, H1, pulse area, and pulse power volume all increased significantly at the left chon position in the PC group compared to the TE group (Figures 9, 10, 11). These results suggest that the increases in $\mathrm{H} 1$, pulse area, and pulse power volume at the left chon position may be caused by the features of the pericardium or by the effects of SA-acu-PC.

On the other hand, H1, pulse area, and pulse power volume decreased at the left chon in the TE group compared to the PC group (Figures 9, 10, 11). According to Oriental medical physiology, the pericardium and the triple energizer are coupled by Yin-Yang exterior-interior relationship, thus, their functions are common in some aspects, while opposed in other aspects. For instance, the pericardium and the triple energizer both pertain ministerial fire, while the pericardium governs blood circulation, whereas the triple energizer governs qi transformation.

In this study, the changes in H1, pulse area, and pulse power volume at the left chon in the TE group were mostly the opposite to those in the PC group. Therefore, it can be suggested that the changes in $\mathrm{H} 1$, pulse area, and pulse power volume at the left chon may be considered as possible indicators for investigating the different properties of the pericardium and the triple energizer, and also the different effects of SA-acu-PC and SA-acu-TE.

In addition to the left chon, $\mathrm{H} 1$ increased significantly at the left gwan in the PC group compared to the TE group (Figure 9). The left gwan corresponds to the liver, and the foot reverting yin liver meridian correlates with the hand reverting yin pericardium meridian. It is assumed that SA-acu-PC influenced not only the pericardium but also the liver which is related to the pericardium.

With regard to triple energizer jung-gyuck (SA-acu-TE), the pulse power volume significantly decreased at the right chon and the right gwan in the TE group compared to the control group (Figure 11). Moreover, the main peak angle (MPA) and radial augmentation index (RAI) increased significantly at 
the right gwan in the TE group compared to the control group or the PC group (Figures 12, 13).

From these results, it may be postulated that the decrease in pulse power volume at the right chon and the right gwan, and the increases in RAI and MPA at the right gwan may be caused by the features of the triple energizer or by the effect of SA-acu-TE.

In the theory of Oriental medical diagnosis, the left pulse is related to blood and the right pulse is related to qi. As mentioned earlier, the pericardium supports blood circulation, while the triple energizer governs qi transformation. Thus, the triple energizer is more likely to correspond to right side of the radial pulse than to the left side.

The triple energizer is also called the triple burner, because it burns in three areas of the body, upper, middle, and lower. The upper burner warms the heart and lungs, the middle burner warms the spleen and stomach, and the lower burner warms the liver and kidneys. It might be difficult, therefore, to determine the corresponding pulse position for the triple energizer among the three positions of chon, gwan, and cheock. However, taking into account the overall results of this study, the effects of SA-acu-TE seem to appear mostly at the right gwan.

Since the effects of SA-acu-TE appeared mainly at the right gwan, it is also possible to assume that the effects of SA-acu-TE appeared mainly on the right gwan because the technique of SA-acu-TE might have affected the middle energizer more than the upper or lower energizers, even though the upper, middle, and lower energizers correspond to the right chon, gwan, and cheock respectively.

As for the correspondences of internal organs to pulse positions, the pericardium and the triple energizer have no corresponding positions in terms of the general principles of Oriental medical pulse diagnosis.

According to the general correspondences, the deep level of the left-chon corresponds to the heart, and the superficial level of the left-chon corresponds to the small intestine which is in Yin-Yang exterior-interior relationship with the heart. However, several classics such as 『Zhang Jie bing's complete

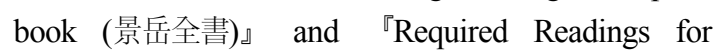
Medical Professionals (醫宗必讀)』correlate the deep level of the left-chon with the heart ${ }^{17)}$, and the superficial level of the left-chon with the pericardium which is the outer shield of the heart ${ }^{18)}$. Both opinions are prima facie theoretically reasonable. However, the results of the current study seem to agree with the latter, in that H1, pulse area, and pulse power volume increased at the left chon position by SA-acu-PC.

In addition, since the triple energizer regulates qi transformation and burns fire in the upper, middle, and lower body cavities, it may correspond to all the three positions of chon, gwan, and cheock on the right-hand side. However, the present study shows that the right gwan responded most when stimulated with SA-acu-TE. It remains uncertain whether the right gwan is the only corresponding position for the triple energizer, or whether the right gwan responds only in case of Sa-Am acupuncture and the right chon or the right cheock would respond in other cases.

The correspondences of the pericardium and the triple energizer to pulse positions is not clear yet. Further studies on this issue will be needed in the future.

Human body maintains homeostasis through various mechanisms, and it is natural that changes in human body induced by external stimulus tend recover back to the previous states gradually over time. In the present study, the changes in pulse parameters mostly appeared right after acupuncture and diminished gradually over time. However, some parameters started to change with some time interval after acupuncture, and some changes were magnified even more over time.

For instance, no significant change in $\mathrm{H} 1$ at the 
position of left gwan was found right after nor 30 min after SA-acu-PC, but a significant increase was found $60 \mathrm{~min}$ after SA-acu-PC (Figure 9). As mentioned earlier, the left gwan corresponds to the liver, and the foot reverting yin liver meridian correlates with the hand reverting yin pericardium meridian. We assumed that SA-acu-PC influenced not only the pericardium but also the liver which is related to the pericardium, and it might take some time to transfer the influence from the corresponding organ and meridian to the related organs and meridians.

In addition, the main peak angle at the right gwan position increased significantly right after SA-acu-TE, and then increased even more $60 \mathrm{~min}$ after SA-acu-TE (Figure 12). We assume that there may exist a mechanism to amplify the therapeutic stimulus to achieve the desired therapeutic effect in human body. It may be how a tenuous stimulus by a fine acupuncture needle induces a tremendous therapeutic effect to treat disease.

The present study was carried out on healthy subjects, using Sa-am acupuncture with pericardium jung-gyuck, and triple energizer jung-gyuck, and analyzed the parameters mainly of the representative pulse wave gained from the central transducer. Further studies are expected to be conducted on subjects with various conditions, using various acupuncture techniques other than Sa-Am acupuncture, or various specific techniques of Sa-Am acupuncture other than jung-gyuck (such as seung-gyuck, han-gyuck and yul-gyuck), and analyzing various pulse parameters from all pulse waves at different pressure levels.

\section{Conclusion}

The effects of acupuncture on the pulse were investigated using Sa-Am acupuncture with pericardium jung-gyuck (SA-acu-PC) and Sa-Am acupuncture with triple energizer jung-gyuck
(SA-acu-TE) in sixty healthy volunteers and the results were as follows.

1. The changes observed in periodic parameters may be considered as the common effects of both SA-acu-TE and SA-acu-PC. SA-acu-TE was more effective than SA-acu-PC in these effects.

2. The increases in pulse amplitude, pulse area, and pulse power volume at the left chon may imply the features of the pericardium and the effects of SA-acu-PC.

3. The decrease in pulse power volume, and the increases in radial augmentation index and main peak angle may imply the features of the triple energizer and the effects of SA-acu-TE.

4. The effects of SA-acu-PC appeared mainly at the left chon position, and the effects of SA-acu-TE appeared mainly at the right gwan position.

\section{References}

1. Li C, Jin JP. New bilingual edition of Introduction to Medicine. $1^{\text {st }}$ ed. Seoul:bubinbooks. 2009: 397-400.

2. Physiology Compilation Committee of Korean Medical Colleges. The Physiology of Oriental Medicine. $1^{\text {st }}$ ed. Paju:Jipmoon. 2008:327-330, 338-344.

3. Classics Compilation Committee of Korean Medical Colleges. Classified Internal Classic. $2^{\text {nd }}$ ed. Daejeon:Juminbooks. 2006:270-271.

4. Song JC, Kwon KB, Lee SY, Lee HJ, Ryu DG. Study On Shimpo Samcho and Myoungmoon In Oriental Medicine. Korean Journal Oriental Physiology \& Pathology. 2007;21(2):362-369.

5. World Health Organization. WHO international standard terminologies on traditional medicine in the western pacific region. 2007:233, 235.

6. Meridians \& Acupoints Compilation Committee of Korean Medical Colleges. Principles of Meridians \& Acupoints; A Guidebook for 
College Students. $6^{\text {th }}$ ed. Daejeon:Jongryeonamu Publishing Co. 2012:349.

7. Yim YK, Kang HJ, Lee BR, Yang GY, Lee H, Kim KC. The Effect of FoodIntakes on Radial Pulse Amplitude. Korean Journal of Acupuncture. 2011;28(2):13-22.

8. Yim YK, Park KS. A study on the Effect of Food Intakeon Radial Pulse using Fourier Analysis. The Journal of Korean Oriental Medicine. 2011;32(4):139-148.

9. Yim YK, Lee C, Lee HJ, Park KS. Gender and Measuring-position Differences in the Radial Pulse of Healthy Individuals. Journal of Acupuncture and Meridian Studies. 2014;7(6): 324-330

10. Yim YK, Lee H, Lee BR, Lee YH, Yoon YJ, Ro JY. Effect of Sa-Am Acupuncture on Radial Pulse ; A Comparative Study of Stomach -tonification and Spleen-tonification. Korean Journal of Acupuncture. 2011;28(3): 25-32.

11. Yim YK. Sa-Am Acupuncture with Spleen Seung-gyuck induces an acute increase of radial pulse parameters related to arterial stiffness in healthy subjects. The Journal of Korean Oriental Medicine. 2014;35(2):19-27.

12. Hugh MP, Douglas GA, Richard HS, Li YP, Wu TX, Adrian W, et al. Revised STandards for
Reporting Interventions in Clinical Trials of Acupuncture (STRICTA): Extending the CONSORT Statement. PLOS medicine. 2010;7(6):1-11.

13. Meridians \& Acupoints Compilation Committee of Korean Medical Colleges. Practice of Acupuncture ; A Guidebook for College Students. Daejeon:Jongryeonamu Publishing Co. 2015:55.

14. Lee SK, Park WH. A Study on the Cun, Guan, Chi (Inch, Bar, Cubit) from the Elementary Course For Medicine(Yi Xue Ru Men). The Journal of the Society of Korean Medicine Diagnostics. 2005;9(2):10-24.

15. Kim YJ, Kang JH, Yoon KS, Jo SE, Hong SJ, Yun GW, et al. The Effects of Gall Bladder Jeonggyeok and Seunggyeok on Radial Pulse Variation in Healthy Human Subjects. The Acupuncture. 2016;33(3):9-62.

16. Yim YK, Park KS. The effect of Short-term Bed-rest on Radial Pulse in Healthy Subjects. Journal of Acupuncture and Meridian Studies. 2014;7(3):133-139.

17. Jeong CY, Yoon CY. A study on chon gwan cheock of chongu pulse dignosis corresponding to organs. Journal of Korean Medical classics. 2008;21(4):29-40.

18. Kaptchuk TJ. The web that has no weaver. New York:McGraw-Hill. 2000:90-7, 194-210, 323-5. 